\title{
Cardiac Surgery in Germany during 2012: A Report on Behalf of the German Society for Thoracic and Cardiovascular Surgery
}

\author{
Andreas Beckmann ${ }^{1}$ Anne-Kathrin Funkat ${ }^{2}$ Jana Lewandowski ${ }^{1}$ Michael Frie ${ }^{3}$ Wolfgang Schiller ${ }^{4}$ \\ Khosro Hekmat ${ }^{5}$ Jan F. Gummert ${ }^{6}$ Friedrich Wilhelm Mohr ${ }^{2}$
}

${ }^{1}$ German Society for Thoracic and Cardiovascular Surgery, Langenbeck-Virchow-Haus, Berlin, Germany

${ }^{2}$ Department of Cardiac Surgery, Herzzentrum, University of Leipzig, Leipzig, Germany

${ }^{3}$ FOM Hochschule fuer Oekonomie and Management, Essen, Germany

${ }^{4}$ Department of Cardiac Surgery, University Bonn, Bonn, Germany

${ }^{5}$ Department of Cardiothoracic Surgery, University of Cologne,

Cologne, Germany

${ }^{6}$ Clinic for Thoracic and Cardiovascular Surgery, Heart and Diabetes

Center NRW, Bad Oeynhausen, Germany

\begin{abstract}
Address for correspondence Dr. Andreas Beckmann, Deutsche Gesellschaft für Thorax-, Herz- und Gefäßchirurgie [DGTHG], Langenbeck-Virchow-Haus, Luisenstr. 58-59, 10117 Berlin, Germany (e-mail: gf@dgthg.de).
\end{abstract}

Thorac Cardiovasc Surg 2014;62:5-17.

\author{
Abstract \\ Keywords \\ - cardiac surgery \\ - outcomes \\ - registry \\ - heart valve disease \\ - coronary heart \\ disease \\ - congenital heart \\ disease \\ - aortic surgery \\ - heart rhythm \\ disorders \\ - organ transplantation
}

On the basis of a voluntary registry of the German Society for Thoracic and Cardiovascular Surgery (GSTCVS), data of all cardiac surgical procedures performed in 79 German cardiac surgical units during the year 2012 are presented. In 2012, a total of 98,792 cardiac surgical procedures (ICD and pacemaker procedures excluded) were submitted to the registry. More than $13.8 \%$ of the patients were older than 80 years, which is a further increase in comparison to previous years. In-hospital mortality in 42,060 isolated coronary artery bypass grafting procedures ( $84.6 \%$ on-pump and $15.4 \%$ off-pump) was $2.9 \%$. In 28,521 isolated valve procedures (including 6,804 catheter-based procedures), an in-hospital mortality of $4.8 \%$ was observed. This long-lasting registry of the GSTCVS will continue to be an important tool for quality control and voluntary public reporting by illustrating current facts and developments of cardiac surgery in Germany.

\section{Introduction}

Increasing demands for quality assurance in medicine-by patients, relatives, insurance companies, and authorities all over the world-have stimulated the development of a wide range of registries and other tools to answer those needs. As early as in 1978, the board of directors of the German Society for Thoracic and Cardiovascular Surgery (www.dgthg.de) decided to set up an annually updated database of all cardiac surgical procedures in terms of a voluntary registry. Since 1989 , the updated data of the registry are published annually. ${ }^{1-22}$ The aim of this registry continues to illustrate developments and trends in cardiac surgery in Germany and it enables each participating cardiac surgical unit to compare its own results with the nationwide achievements.

For monitoring actual conditions as well as the development in cardiac medicine, the registry includes particular techniques such as off-pump cardiac surgery or minimal received

October 18, 2013

accepted

October 21, 2013 (c) 2014 Georg Thieme Verlag KG Stuttgart · New York
DOI http://dx.doi.org/ 10.1055/s-0033-1361953. ISSN 0171-6425. 
invasive mitral valve operations and also innovative technologies such as transapical or transvascular aortic valve implantation (TAVI) (see - Table V3). Thereby important findings for present patient safety and the future of patient care may be collected and evaluated.

The data presented in this report comprehend assorted data of the year 2012 .

\section{Materials and Methods}

Since 2004, a standardized questionnaire gathers detailed information about each individual procedure exactly defined by a German adaption of the International Classification of Procedures in Medicine called operation code (Operationenund Prozedurenschlüssel).

All participants were requested to complete the structured questionnaire until January 21, 2013, asking for all performed procedures and associated in-hospital mortality. The completed questionnaire had to be sent to the office of the GSTCVS, where they were evaluated for completeness and compiled for further analysis, thus ensuring anonymity for each individual institution. This compilation algorithm guarantees a high compliance for submission of complete datasets.

Inclusion criteria for the registry 2012 were all patients receiving cardiac surgical procedures performed between January 1, 2012, and December 31, 2012, unrelated to the dates of admission or discharge as compared with other registries. Alike to all previous years, the number of procedures was counted rather than individual patients. For example, if a patient required additional coronary artery bypass grafting ( $C A B G$ ) due to a complication after initial aortic valve replacement during one admission, one count in the category "aortic valve replacement" and another in the category "coronary surgery" are enumerated. Thus, the registry contains more procedures than the real number of patients operated on.

Death of patients was defined as in-hospital mortality. As per the definition, the observed mortality is always attributed to the first cardiac procedure, for example, the death of a patient requiring $C A B G$ due to a complication of an aortic valve procedure would only be attributed to the aortic valve procedure.

The main reason for this structural setup of the registryestablished over several decades-is to keep the German data privacy act with its specific regulations for patients. Furthermore, it seemed to be relevant getting detailed information about all performed procedures and not only the number of treated patients. Last but not the least, the process of data acquisition had to be simplified for all cardiac surgery units in Germany thus enabling the submission of a complete dataset, regardless of the locally existing hard- and software used for data management.

In 2012, a total of 79 institutions performed heart surgery. Fortunately, all units answered the questionnaire and delivered a complete dataset for the year 2012 including hospital mortality rates.

\section{Registry Data 2012}

-Table 1 illustrates the development of procedures using extracorporeal circulation (ECC) over more than two decades in Germany. The number of heart operations using ECC remains on a stable level.

Overall, 177,694 procedures were reported to the registry for the year 2012, an increase of 2.5\% (2011: 173,347 procedures). A total of 101,887 cardiac surgical procedures (excluded: ICD, pacemakers, and miscellaneous procedures without ECC) display an increase of $1.57 \%(n=1,596)$ compared with the year 2011 (100,291 procedures) (-Table 2). - Tables 3 to 6, - Tables V1 to 7, - Tables C1 to C3, - Tables Con1 and Con2, - Tables Mis1 to 5, and - Figs. 1 to 9 demonstrate the compiled registry data of 2012 for various categories.

Compared with the data of previous years, several important developments continue unchanged in 2012. Over nearly

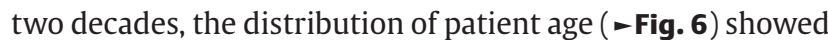
a shift to older patients with presently $53.7 \%$ of the cardiac procedures performed in patients of at least 70 years of age and $13.8 \%$ in patients of 80 years or older. However, mortality remained on the same low level or even decreased slightly over the represented decades (see - Fig. 1B). The rate of CABG procedures decreased over the past years while the relative number of off-pump CABG showed a slight increase to $15.4 \%$ (2011: 14.7\%) (-Fig. 3).

Since 2004 more than $50 \%$ of isolated mitral valve procedures are reconstructions, in 2012 mitral valve reconstruction could be achieved in $64.4 \%$ of the procedures (-Fig. 8). On the basis of the fact that all isolated mitral valve procedures are included, regardless of the underlying disease, valve morphology, or urgency of operation, it has to be assumed that the relative rate of mitral valve reconstruction would certainly be even higher if patients without possibility or indication for reconstruction would have been excluded (e.g., mitral valve stenosis, calcifications, or endocarditis). In other publications, for example Gammie et $a,^{23}$ patients with

Table 1 Frequency of open heart procedures in Germany from 2003 to 2012

\begin{tabular}{|l|l|l|l|l|l|l|l|l|l|l|}
\hline Year & $\mathbf{2 0 0 3}$ & $\mathbf{2 0 0 4}$ & $\mathbf{2 0 0 5}$ & $\mathbf{2 0 0 6}$ & $\mathbf{2 0 0 7}$ & $\mathbf{2 0 0 8}$ & $\mathbf{2 0 0 9}$ & $\mathbf{2 0 1 0}$ & $\mathbf{2 0 1 1}$ & $\mathbf{2 0 1 2}$ \\
\hline Total number of units & 78 & 79 & 79 & 80 & 80 & 79 & 80 & 79 & 78 & 79 \\
\hline Total number of operations & 94,712 & 96,340 & 91,967 & 91,057 & 91,618 & 89,773 & 86,916 & 84,686 & 84,402 & 84,388 \\
\hline Average per unit & 1,214 & 1,219 & 1,164 & 1,138 & 1,145 & 1,136 & 1,086 & 1,072 & 1,082 & 1,068 \\
\hline
\end{tabular}


Table 2 Results of all 79 units performing cardiac surgery in 2012

\begin{tabular}{|l|l|l|l|l|}
\hline Category & With ECC & Without ECC & Total & change \\
\hline Valve procedures & 21,918 & 6,603 & 28,521 & $+5.7 \%$ \\
\hline Coronary surgery & 48,443 & 6,859 & 55,302 & $+0.0 \%$ \\
\hline Congenital heart surgery & 4,651 & 942 & 5,593 & $-0.1 \%$ \\
\hline Surgery of thoracic aorta & 6,805 & 575 & 7,380 & $+2.5 \%$ \\
\hline Other cardiac surgery & 1,276 & 1,197 & 2,473 & $-6.0 \%$ \\
\hline Assist devices & 812 & 1,806 & 2,618 & $+24.1 \%$ \\
\hline Pacemaker and ICD & 93 & 25,139 & 25,232 & $-3.0 \%$ \\
\hline Extracardiac surgery & 390 & 50,185 & 50,575 & 177,694 \\
\hline Total & 84,388 & 93,306 & $+6.4 \%$ \\
\hline
\end{tabular}

Abbreviation: ECC, extracorporeal circulation.

Note: The percentage indicates changes compared with 2011.

Table 3 Distribution of individual units according to the number of cardiac surgery procedures with or without ECC

\begin{tabular}{|l|l|l|l|l|l|}
\hline Number of operations & $<500$ & $500-999$ & $1,000-1,499$ & $1,500-1,999$ & $2,000-5,000$ \\
\hline Number of units & 6 & 24 & 26 & 13 & 10 \\
\hline Average per unit & 378 & 803 & 1,186 & 1,706 & 2,781 \\
\hline Min-max & $271-474$ & $538-990$ & $1,019-1,468$ & $1,517-1,959$ & $2,169-3,814$ \\
\hline
\end{tabular}

Abbreviation: ECC, extracorporeal circulation.

Note: One unit only performs operations in patients with congenital heart disease.

Table 4 Distribution of units according to surgical profiles in 2012

\begin{tabular}{|l|l|}
\hline Procedures & Number of units \\
\hline Coronary surgery & 78 \\
\hline Valve surgery & 78 \\
\hline Congenital heart surgery under use of ECC in children $<1 \mathrm{y}$ & $24^{\mathrm{a}}$ \\
\hline Heart transplantation & $22^{\mathrm{b}}$ \\
\hline Heart-lung transplantation & 4 \\
\hline
\end{tabular}

Abbreviation: ECC, extracorporeal circulation.

a Surgery for congenital heart disease with ECC in children $<1$ year $(n=2,024)$; thereof: $1-19$ operations in 3 units, $21-45$ operations in 7 units, 50 90 operations in 6 units, and 109-269 operations in 8 units.

${ }^{b}$ Heart transplantations ( $\left.\mathrm{HTx}\right)(n=327)$ : $64 \%$ of the HTx in 2012 were performed by 6 units with $\geq 15$ HTx per year; thereof: 1-4 transplantations in 4 units, 5-7 transplantations in 6 units, 10-14 transplantations in 6 units, and 21-73 transplantations in 6 units.

Table 5 Additional demographic data for procedures with ECC in 2012 and 2011

\begin{tabular}{|l|l|l|l|l|}
\hline & \multicolumn{2}{|c|}{2012} & \multicolumn{2}{|c|}{2011} \\
\hline Emergency operations & 11,878 & $11.6 \%$ & 11,911 & $11.9 \%$ \\
\hline Redo procedures & 8,424 & $8.2 \%$ & 8,511 & $8.5 \%$ \\
\hline Age $>69 \mathrm{y}$ & 97,572 & $53.7 \%$ & 95,456 & $53.6 \%$ \\
\hline
\end{tabular}

Abbreviation: ECC, extracorporeal circulation.

Note: The numbers in each category reflect procedures and not individual patients. 
8 Cardiac Surgery in Germany during 2012 Beckmann et al.

Table 6 Gender distribution

\begin{tabular}{|l|l|l|}
\hline \multicolumn{3}{|l|}{ Male/female ratio among cardiac procedures } \\
\hline Valve procedures & $56 \%$ & $44 \%$ \\
\hline Coronary surgery & $76 \%$ & $24 \%$ \\
\hline Congenital heart surgery & $53 \%$ & $47 \%$ \\
\hline Surgery of thoracic aorta & $69 \%$ & $31 \%$ \\
\hline Other cardiac surgery & $52 \%$ & $48 \%$ \\
\hline Assist devices & $77 \%$ & $23 \%$ \\
\hline Pacemaker and ICD & $65 \%$ & $35 \%$ \\
\hline Extracardiac surgery & $63 \%$ & $37 \%$ \\
\hline Total & $66 \%$ & $34 \%$ \\
\hline
\end{tabular}

Note: Coronary surgery (48,443 on-pump and 6,859 off-pump procedures) and all congenital heart surgery procedures are included in this table.

mitral valve stenosis, endocarditis, and emergency procedures were excluded, so the reconstruction rate must be interpreted with caution compared to this registry.

The continued increase of left ventricular assist device implantation (-Fig. 10) emphasizes the increasing relevance of mechanical circulatory support.

Again the most remarkable trend is the repeated extensive increase of TAVI procedures ( $\mathbf{F i g . 5}$ ), although the number of isolated aortic valve replacement procedures remained on a stable level. Starting in 2006 with just 78 implantations ( $0.67 \%$ of isolated aortic valve procedures), in 2012 a total of 6,479 (35.5\%) TAVI were reported to the registry in 2012. It must be emphasized that the 79 institutions which contribute their data to this registry do not represent all departments performing TAVI in Germany. In addition, there are some institutions performing TAVI via transvascular access without a cardiac surgery on-site. This proceeding is not in accordance with the recommendations of the European guideline on the management of valvular heart disease version 2012.24

In this context, the short-, mid-, and long-term results of the German Aortic Valve Registry and the annual findings of the legal quality assurance ( $\$ 137$ SGB V), conducted by the AQUA Institute, are of outstanding patient benefit.

Table V1 Isolated heart valve procedures

\begin{tabular}{|l|l|l|l|}
\hline Procedures & $\boldsymbol{n}$ & Deaths & $\%$ \\
\hline Single & 18,094 & 624 & 3.4 \\
\hline Double & 3,133 & 289 & 9.2 \\
\hline Triple & 400 & 52 & 13.0 \\
\hline Single, transcatheter access & 6,795 & 382 & 5.6 \\
\hline Double, transcatheter access & 9 & 2 & 22.2 \\
\hline Not specified & 90 & 7 & 7.8 \\
\hline Total & 28,521 & 1,356 & 4.8 \\
\hline
\end{tabular}

Notes: Combined procedures (with CABG, aortic surgery) are excluded. Transcatheter procedures: 6,479 aortic valve implantations; 42 mitral valve implantations; 269 mitral valve repairs, 5 tricuspidal valve repairs, 9 double aortic and mitral valve procedures, and no pulmonary valve implantation.
Table V2 Access type in single valve procedures

\begin{tabular}{|l|l|l|l|}
\hline Position & $\boldsymbol{n}$ & Deaths & $\%$ \\
\hline Aortic sternotomy & 9,738 & 314 & 3.2 \\
\hline Aortic part. sternotomy & 2,168 & 36 & 1.7 \\
\hline Aortic transvascular & 3,550 & 167 & 4.7 \\
\hline Aortic transapical & 2,929 & 210 & 7.2 \\
\hline Mitral sternotomy & 3,110 & 186 & 6.0 \\
\hline Mitral mis & 2,512 & 42 & 1.7 \\
\hline Mitral transcatheter & 311 & 4 & 1.3 \\
\hline Tricuspid sternotomy & 407 & 36 & 8.8 \\
\hline Tricuspid mis & 109 & 7 & 6.4 \\
\hline Tricuspid transcatheter & 5 & 1 & 20.0 \\
\hline Pulmonary sternotomy & 50 & 3 & 6.0 \\
\hline Pulmonary mis & 0 & - & - \\
\hline Pulmonary transcatheter & 0 & 0 & - \\
\hline Total & 24,889 & 1,006 & 4.0 \\
\hline
\end{tabular}

Notes: A total of 2,512 (45\%) mitral valve procedures were performed by a minimally invasive access. The number of isolated aortic valve procedures by sternotomy increased from 11,668 procedures in 2011 to 11,906 in 2012.

Table V3 Isolated aortic valve procedures

\begin{tabular}{|l|l|l|l|}
\hline Type & $\boldsymbol{n}$ & Deaths & \% \\
\hline Mechanical prosthesis & 1652 & 37 & 2.2 \\
\hline Xenograft & 10091 & 308 & 3.1 \\
\hline Homograft & 36 & 4 & 11.1 \\
\hline Reconstruction & 127 & 1 & 0.8 \\
\hline Total & 11906 & 350 & 2.9 \\
\hline
\end{tabular}

Notes: Of 11,906 procedures, 2168 (18\%) were performed via partial sternotomy. Transcatheter procedures were not included.

Table V4 Isolated mitral valve procedures

\begin{tabular}{|l|l|l|l|}
\hline Type & $\boldsymbol{n}$ & Deaths & \% \\
\hline Mechanical prosthesis & 570 & 38 & 6.7 \\
\hline Xenograft & 1,425 & 131 & 9.2 \\
\hline Homograft & 6 & 0 & 0.0 \\
\hline Reconstruction & 3,621 & 59 & 1.6 \\
\hline Total & 5,622 & 228 & 4.1 \\
\hline
\end{tabular}

Notes: Of 5,622 procedures, 2,512 (45\%) were performed via minimally invasive access. Transcatheter procedures were excluded. 
Table V5 Multiple heart valve procedures

\begin{tabular}{|l|l|l|l|}
\hline Combination & $\boldsymbol{n}$ & Deaths & $\%$ \\
\hline Aortic + mitral & 1,345 & 165 & 12.3 \\
\hline Mitral + tricuspid & 1,489 & 103 & 6.9 \\
\hline Aortic + tricuspid & 185 & 18 & 9.7 \\
\hline Tricuspid + pulmonary & 16 & 1 & 6.3 \\
\hline Aortic + pulmonary & 98 & 2 & 2.0 \\
\hline Aortic + mitral + tricuspid & 399 & 52 & 13.0 \\
\hline Aortic + mitral + pulmonary & 1 & 0 & 0.0 \\
\hline Total & 3,533 & 341 & 9.7 \\
\hline
\end{tabular}

Note: Transcatheter procedures were excluded.

ancluding Ross procedures.

\section{Discussion}

The registry of the GSTCVS enables a comprehensive overview of all cardiac surgical procedures performed in Germany in 2012. The accuracy of this registry is considered to be high due to the implemented compilation algorithm using operation codes. This is supported by other authors who could demonstrate a high accuracy for major outcome parameters in unaudited registries. ${ }^{25}$ In continuation to previous years, it can be concluded that cardiac surgery in Germany is performed on a constantly high level with a low in-hospital mortality compared with other international registries. This conclusion is especially important in an era of demographic change of the German population resulting in a continuously

Table V6 Mitral valve surgery-implantation resp. replacement versus reconstruction

\begin{tabular}{|c|c|c|c|c|c|c|c|c|c|c|}
\hline \multirow{2}{*}{$\begin{array}{l}\text { Mitral valve } \\
\text { surgery }\end{array}$} & \multirow[t]{2}{*}{$n$} & \multirow{2}{*}{$\begin{array}{l}\text { Total } \\
\text { deaths }\end{array}$} & \multirow[t]{2}{*}{ \% death } & \multirow[t]{2}{*}{$\%$ reconstruction } & \multicolumn{2}{|c|}{ Replacement } & \multirow{2}{*}{$\begin{array}{l}\% \\
\text { death }\end{array}$} & \multicolumn{2}{|c|}{ Reconstruction } & \multirow[t]{2}{*}{$\%$ death } \\
\hline & & & & & $n$ & Deaths & & $n$ & Deaths & \\
\hline Isolated & 5,622 & 228 & 4.1 & 64.4 & 2,001 & 169 & 8.4 & 3,621 & 59 & 1.6 \\
\hline \multicolumn{11}{|l|}{ Mitral valve +} \\
\hline Aortic valve & 1,345 & 165 & 12.3 & 42.5 & 773 & 115 & 14.9 & 572 & 50 & 8.7 \\
\hline $\begin{array}{l}\text { Tricuspid valve } \\
\text { reconstruction }\end{array}$ & 1,445 & 99 & 6.9 & 65.3 & 501 & 54 & 10.8 & 944 & 45 & 4.8 \\
\hline CABG & 2,640 & 252 & 9.5 & 70.2 & 786 & 129 & 16.4 & 1,854 & 123 & 6.6 \\
\hline $\begin{array}{l}\text { CABG + aortic } \\
\text { valve replacement }\end{array}$ & 674 & 119 & 17.7 & 53.1 & 316 & 68 & 21.5 & 358 & 51 & 14.2 \\
\hline Total & 11,726 & 863 & 7.4 & 62.7 & 4,377 & 535 & 12.2 & 7,349 & 328 & 4.5 \\
\hline
\end{tabular}

Abbreviation: CABG, coronary artery bypass grafting.

${ }^{a}$ Forty-four procedures (not specified mitral valve + tricuspid valve surgery) were excluded. Deaths: 9.1\% (4/44).

Table V7 Transcatheter valve procedures: $45.2 \%$ of TAVI were performed by transapical access

\begin{tabular}{|c|c|c|c|c|c|c|c|}
\hline & Total & Deaths & Death \% & & ECC & Wit & ECC \\
\hline & & & & $n$ & Deaths & $n$ & Deaths \\
\hline Aortic valve implantation & 6,479 & 377 & 5.8 & 182 & 62 & 6,297 & 315 \\
\hline Transvascular access ${ }^{a}$ & 3,550 & 167 & 4.7 & 80 & 24 & 3,470 & 143 \\
\hline Transapical access & 2,929 & 210 & 7.2 & 102 & 38 & 2,827 & 172 \\
\hline Mitral valve & 311 & 4 & 1.3 & 16 & 1 & 295 & 3 \\
\hline Repair & 269 & 4 & 1.5 & 11 & 1 & 258 & 3 \\
\hline Implantation $^{\mathrm{b}}$ & 42 & 0 & 0.0 & 5 & 0 & 37 & 0 \\
\hline Tricuspid valve repair & 5 & 1 & 20.0 & 2 & 0 & 3 & 1 \\
\hline Aortic + mitral valve implantation & 9 & 2 & 22.2 & 1 & 0 & 8 & 2 \\
\hline Aortic valve implantation ${ }^{\mathrm{b}}+\mathrm{CABG}$ & 23 & 5 & 21.7 & 3 & 2 & 20 & 3 \\
\hline Total & 6,827 & 389 & 5.7 & 204 & 65 & 6,623 & 324 \\
\hline
\end{tabular}

Abbreviations: CABG, coronary artery bypass grafting; ECC, extracorporeal circulation; TAVI, transapical or transvascular aortic valve implantation. Notes: Pulmonary valve implantation for congenital heart disease are excluded, no procedure was reported for adults without congenital lesion. $2.8 \%$ of TAVI procedures were performed under use of ECC. It has to be assumed that ECC was mostly used in emergency situations, which explains the mortality of $34.1 \%$ in this group. Nevertheless, this underlines the necessity of a cardiac surgery on-site for TAVI procedures.

a Femoral, subclavian, or transaortic access.

${ }^{\mathrm{b}}$ Transvascular and transapical access. 
10 Cardiac Surgery in Germany during 2012 Beckmann et al.

Table C1 Isolated CABG with ECC and combined procedures with ECC

\begin{tabular}{|l|l|l|l|}
\hline Procedures & $n$ & Deaths & $\%$ \\
\hline CABG & \multicolumn{1}{|l|}{42,060} & 1,217 & 2.9 \\
\hline CABG + & \multicolumn{3}{|l|}{} \\
\hline Transmyocardial laser revascularization & 6 & 0 & 0.0 \\
\hline Left ventricular-aneurysm resection & 168 & 11 & 6.5 \\
\hline Aortic valve replacement & 8,216 & 432 & 5.3 \\
\hline Transcatheter aortic valve implantation & 23 & 5 & 21.7 \\
\hline Mitral valve replacement & 786 & 129 & 16.4 \\
\hline Mitral valve repair & 1,854 & 123 & 6.6 \\
\hline Aortic + mitral valve replacement & 316 & 68 & 21.5 \\
\hline Aortic valve replacement + mitral valve repair & 358 & 51 & 14.2 \\
\hline Other & 1,515 & 95 & 6.3 \\
\hline Total & 55,302 & 2,131 & 3.9 \\
\hline
\end{tabular}

Abbreviations: CABG, coronary artery bypass grafting; ECC, extracorporeal circulation.

increasing patient age and related comorbidities, both leading to a higher preoperative risk profile.

Compared with 2011, the number of cardiac surgery procedures nearly remains on the same level due to the still increasing number of catheter-based valve procedures.

Table C2 Isolated CABG with ECC

\begin{tabular}{|l|l|l|l|}
\hline Number of grafts & $N$ & Deaths & $\%$ \\
\hline Single & 1,213 & 73 & 6.0 \\
\hline Double & 7,399 & 250 & 3.4 \\
\hline Triple & 15,667 & 466 & 3.0 \\
\hline Quadruple & 8,734 & 242 & 2.8 \\
\hline Quintuple + more & 2,560 & 57 & 2.2 \\
\hline Total & 35,573 & 1,088 & 3.1 \\
\hline
\end{tabular}

Abbreviations: CABG, coronary artery bypass grafting; ECC, extracorporeal circulation.

Table C3 Isolated CABG off-pump

\begin{tabular}{|l|l|l|l|}
\hline Number of grafts & $\boldsymbol{n}$ & Deaths & \% \\
\hline Single & 1,525 & 33 & 2.2 \\
\hline Double & 1,938 & 45 & 2.3 \\
\hline Triple & 2,132 & 40 & 1.9 \\
\hline Quadruple & 750 & 10 & 1.3 \\
\hline Quintuple + more & 142 & 1 & 0.7 \\
\hline Total & 6,487 & 129 & 2.0 \\
\hline
\end{tabular}

Abbreviation: CABG, coronary artery bypass grafting.
Further improvements in the basic configuration of the registry are necessary to allow a more detailed and particularly a risk-adjusted analysis of the data. However, if significant structural changes of data collection for the registry are conducted, it must be ensured that data compatibility still allows further longitudinal data analysis.

Completeness, validity, and further developments will depend on continued efforts of the GSTCVS in close collaboration with all cardiac surgical units in Germany. This will be of outstanding importance for the contribution to patient safety as well as to facilitate a high quality of cardiac surgery in Germany.

Table Con1 Patient's age distribution for congenital heart surgery

\begin{tabular}{|c|c|c|c|}
\hline Age & $n$ & Deaths & $\%$ \\
\hline \multicolumn{4}{|l|}{ (A) Without ECC } \\
\hline Older than $18 \mathrm{y}$ & 34 & 1 & 2.9 \\
\hline $1-17$ y & 142 & 0 & 0.0 \\
\hline Younger than $1 \mathrm{y}$ & 766 & 20 & 2.6 \\
\hline Total A & 942 & 21 & 2.2 \\
\hline \multicolumn{4}{|l|}{ (B) With ECC } \\
\hline Older than $18 \mathrm{y}$ & 989 & 23 & 2.3 \\
\hline $1-17$ y & 1,638 & 16 & 1.0 \\
\hline Younger than $1 \mathrm{y}$ & 2,024 & 79 & 3.9 \\
\hline Total B & 4,651 & 118 & 2.5 \\
\hline
\end{tabular}

Abbreviation: ECC, extracorporeal circulation. 
Table Con2 Congenital heart surgery with and without ECC

\begin{tabular}{|c|c|c|c|c|c|c|c|c|c|}
\hline \multirow[t]{2}{*}{ Lesion/procedure } & \multicolumn{3}{|c|}{ Age $<1$ y } & \multicolumn{3}{|c|}{ Age $1-17 y$} & \multicolumn{3}{|c|}{ Age $\geq 18 y$} \\
\hline & $n$ & Deaths & $\%$ & $n$ & Deaths & $\%$ & $n$ & Deaths & $\%$ \\
\hline ASD & 51 & 0 & 0.0 & 254 & 0 & 0.0 & 287 & 6 & 2.1 \\
\hline Complete AV canal & 174 & 5 & 2.9 & 62 & 0 & 0.0 & 11 & 0 & 0.0 \\
\hline VSD & 326 & 3 & 0.9 & 112 & 1 & 0.9 & 21 & 0 & 0.0 \\
\hline Fallot tetralogy & 177 & 5 & 2.8 & 45 & 0 & 0.0 & 9 & 0 & 0.0 \\
\hline DORV & 44 & 2 & 4.5 & 12 & 0 & 0.0 & 2 & 0 & 0.0 \\
\hline TGA & 159 & 4 & 2.5 & 3 & 0 & 0.0 & 1 & 0 & 0.0 \\
\hline TGA + VSD & 65 & 0 & 0.0 & 8 & 1 & 12.5 & 0 & 0 & - \\
\hline Truncus arteriosus & 31 & 2 & 6.5 & 5 & 0 & 0.0 & 1 & 0 & 0.0 \\
\hline Fontan & 5 & 0 & 0.0 & 231 & 5 & 2.2 & 10 & 2 & 20.0 \\
\hline Norwood & 187 & 26 & 13.9 & 2 & 0 & 0.0 & 0 & 0 & - \\
\hline Pulmonary valve & 48 & 0 & 0.0 & 225 & 4 & 1.8 & 82 & 0 & 0.0 \\
\hline $\begin{array}{l}\text { Transcatheter } \\
\text { pulmonary valve } \\
\text { implantation }\end{array}$ & 1 & 0 & 0.0 & 6 & 0 & 0.0 & 9 & 1 & 11.1 \\
\hline Aortic valve & 61 & 0 & 0.0 & 209 & 1 & 0.5 & 319 & 7 & 2.2 \\
\hline Ross procedure & 10 & 1 & 10.0 & 26 & 0 & 0.0 & 29 & 0 & 0.0 \\
\hline Mitral valve & 43 & 1 & 2.3 & 93 & 1 & 1.1 & 69 & 5 & 7.2 \\
\hline Tricuspid valve & 44 & 2 & 4.5 & 51 & 0 & 0.0 & 44 & 1 & 2.3 \\
\hline PDA & 286 & 9 & 3.1 & 14 & 0 & 0.0 & 3 & 0 & 0.0 \\
\hline Coarctation & 195 & 1 & 0.5 & 44 & 0 & 0.0 & 6 & 0 & 0.0 \\
\hline Transplantation heart & 6 & 0 & 0.0 & 17 & 1 & 5.9 & 0 & 0 & - \\
\hline Transplantation heart + lung & 0 & 0 & - & 1 & 0 & 0.0 & 0 & 0 & - \\
\hline Transplantation lung & 0 & 0 & - & 9 & 1 & 11.1 & 0 & 0 & - \\
\hline Others & 877 & 53 & 6.0 & 351 & 24 & 6.8 & 120 & 3 & 2.5 \\
\hline Total & 2,790 & 114 & 4.1 & 1,780 & 39 & 2.2 & 1,023 & 25 & 2.4 \\
\hline
\end{tabular}

Abbreviations: ASD, atrial septal defect; AV, atrioventricular; DORV, double outlet right ventricle; PDA, patent ductus arteriosus; TGA, transposition at the great arteries; VSD, ventricular septal defect.

Table Mis1 Development of Ross procedures in various age groups

\begin{tabular}{|l|l|l|l|l|l|l|l|}
\hline \multirow{2}{*}{$\begin{array}{l}\text { Autologous aortic valve } \\
\text { replacement (Ross procedure) }\end{array}$} & $\mathbf{2 0 0 2}$ & $\mathbf{2 0 0 5}$ & $\mathbf{2 0 0 8}$ & $\mathbf{2 0 0 9}$ & $\mathbf{2 0 1 0}$ & $\mathbf{2 0 1 1}$ & $\mathbf{2 0 1 2}$ \\
\cline { 2 - 8 } & $\boldsymbol{n}$ & $\boldsymbol{n}$ & $\boldsymbol{n}$ & $\boldsymbol{n}$ & $\boldsymbol{n}$ & $\boldsymbol{n}$ & $\boldsymbol{n}$ \\
\hline Patients $\geq 18 \mathrm{y}$ & 163 & 235 & 207 & 175 & 184 & 134 & 117 \\
\hline Patients $<18 \mathrm{y}$ & 61 & 46 & 42 & 54 & 43 & 40 & 36 \\
\hline Total & 224 & 281 & 249 & 229 & 227 & 174 & 153 \\
\hline
\end{tabular}

Table Mis2 Transplantation all pediatric transplantations (demonstrated in Table Con3) are included in this table

\begin{tabular}{|l|l|l|l|l|l|l|}
\hline \multirow{2}{*}{ Transplantation } & \multicolumn{3}{|c|}{ With ECC } & \multicolumn{3}{c|}{ Without ECC } \\
\cline { 2 - 7 } & $n$ & Deaths & $\%$ & $n$ & Deaths & $\%$ \\
\hline Heart & 327 & 38 & 11.6 & & & \\
\hline Heart + lung & 14 & 1 & 7.1 & & & \\
\hline Lung & 71 & 4 & 5.6 & 244 & 11 & 4.5 \\
\hline
\end{tabular}

Notes: Eurotransplant (ET) reported for the same period 341 heart transplantations (HTx), 13 heart + kidney transplantations, 2 heart + liver transplantations, 10 heart-lung transplantations, 268 double lung, 57 single lung transplantations, 1 lung + kidney transplantations, and 1 lung + liver transplantations. The differences (ET: $-28 \mathrm{LTx},-5 \mathrm{HTx}$ ) may be explained by different inclusion criteria (time of transplantation) for the registry and the ET database. 
12 Cardiac Surgery in Germany during 2012 Beckmann et al.

Table Mis3 Aortic surgery

\begin{tabular}{|c|c|c|c|c|c|c|}
\hline \multirow[t]{2}{*}{ Aortic surgery ${ }^{a}$} & \multicolumn{3}{|c|}{ With ECC } & \multicolumn{3}{|c|}{ Without ECC } \\
\hline & $n$ & Deaths & $\%$ & $n$ & Deaths & $\%$ \\
\hline Supracoronary ascending & 1,623 & 110 & 6.8 & & & \\
\hline Infracoronary ascending & & & - & & & \\
\hline Mechanical valve conduits & 510 & 36 & 7.1 & & & \\
\hline Biological valve conduits & 806 & 79 & 9.8 & & & \\
\hline David technique & 463 & 10 & 2.2 & & & \\
\hline Yacoub technique & 119 & 4 & 3.4 & & & \\
\hline Other & 263 & 27 & 10.3 & & & \\
\hline Supracoronary ascending + aortic valve replacement & 1,363 & 66 & 4.8 & & & \\
\hline Aortic arch replacement ${ }^{\mathrm{b}}$ & 1,472 & 194 & 13.2 & & & \\
\hline Descending & 90 & 6 & 6.7 & 14 & 3 & 21.4 \\
\hline Thoracoabdominal & 89 & 7 & 7.9 & 59 & 7 & 11.9 \\
\hline Endovascular stent, descending & 7 & 0 & 0.0 & 502 & 24 & 4.8 \\
\hline Total & 6,805 & 539 & 7.9 & 575 & 34 & 5.9 \\
\hline
\end{tabular}

Notes: All procedures involving aortic surgery are included. Isolated aortic surgery as well as all possible combined procedures (e.g., additional CABG) are summarized in this category.

aprocedures for abdominal aortic diseases are not included: 486 abdominal and 488 endovascular stents, abdominal.

${ }^{b}$ All possible combined procedures are included in this category; the only common denominator is aortic arch surgery.

Table Mis4 Pacemaker and ICD procedures

\begin{tabular}{|c|c|c|c|c|c|c|c|}
\hline \multirow[t]{2}{*}{ Pacemaker and ICD } & \multirow[t]{2}{*}{ Total } & \multirow[t]{2}{*}{ Deaths } & \multirow[t]{2}{*}{ Death \% } & \multicolumn{2}{|c|}{ With ECC } & \multicolumn{2}{|c|}{ Without ECC } \\
\hline & & & & $n$ & Deaths & $n$ & Deaths \\
\hline Pacemaker: implantation & 9,276 & 54 & 0.6 & 17 & 2 & 9,259 & 52 \\
\hline Pacemaker: exchange & 1,976 & 5 & 0.3 & 0 & 0 & 1,976 & 5 \\
\hline Pacemaker: revision & 2,966 & 38 & 1.3 & 42 & 4 & 2,924 & 34 \\
\hline ICD: implantation & 4,963 & 12 & 0.2 & 1 & 0 & 4,962 & 12 \\
\hline ICD: exchange & 1,812 & 1 & 0.1 & 0 & 0 & 1,812 & 1 \\
\hline ICD: revision & 3,228 & 44 & 1.4 & 29 & 0 & 3,199 & 44 \\
\hline Miscellaneous & 1,011 & 6 & 0.6 & 4 & 0 & 1,007 & 6 \\
\hline Total & 25,232 & 160 & 0.6 & 93 & 6 & 25,139 & 154 \\
\hline
\end{tabular}

Table Mis5 Surgical ablation procedures

\begin{tabular}{|l|l|l|l|}
\hline Energy & Total & Endocardiac ablation, $\boldsymbol{n}$ & Total, $\boldsymbol{n}$ \\
\hline Unipolar radiofrequency & 198 & 159 & 39 \\
\hline Unipolar cryoradiofrequency & 469 & 272 & 197 \\
\hline Bipolar radiofrequency & 1,967 & 263 & 1,704 \\
\hline Cryothermy & 1,706 & 1,439 & 267 \\
\hline Microwave & 38 & 22 & 16 \\
\hline Focused ultrasound & 688 & 71 & 617 \\
\hline Laser & 1 & 0 & 1 \\
\hline other & 11 & 2 & 9 \\
\hline Total & 5,078 & 2,228 & 2,850 \\
\hline
\end{tabular}

Notes: All isolated ablation procedures and all possible combination of procedures (e.g., CABG + ablation) are included. Total of $n=319$ procedures are not specified with regard to endocardiac/epicardiac ablation. 
Cardiac Surgery in Germany during 2012 Beckmann et al. 13

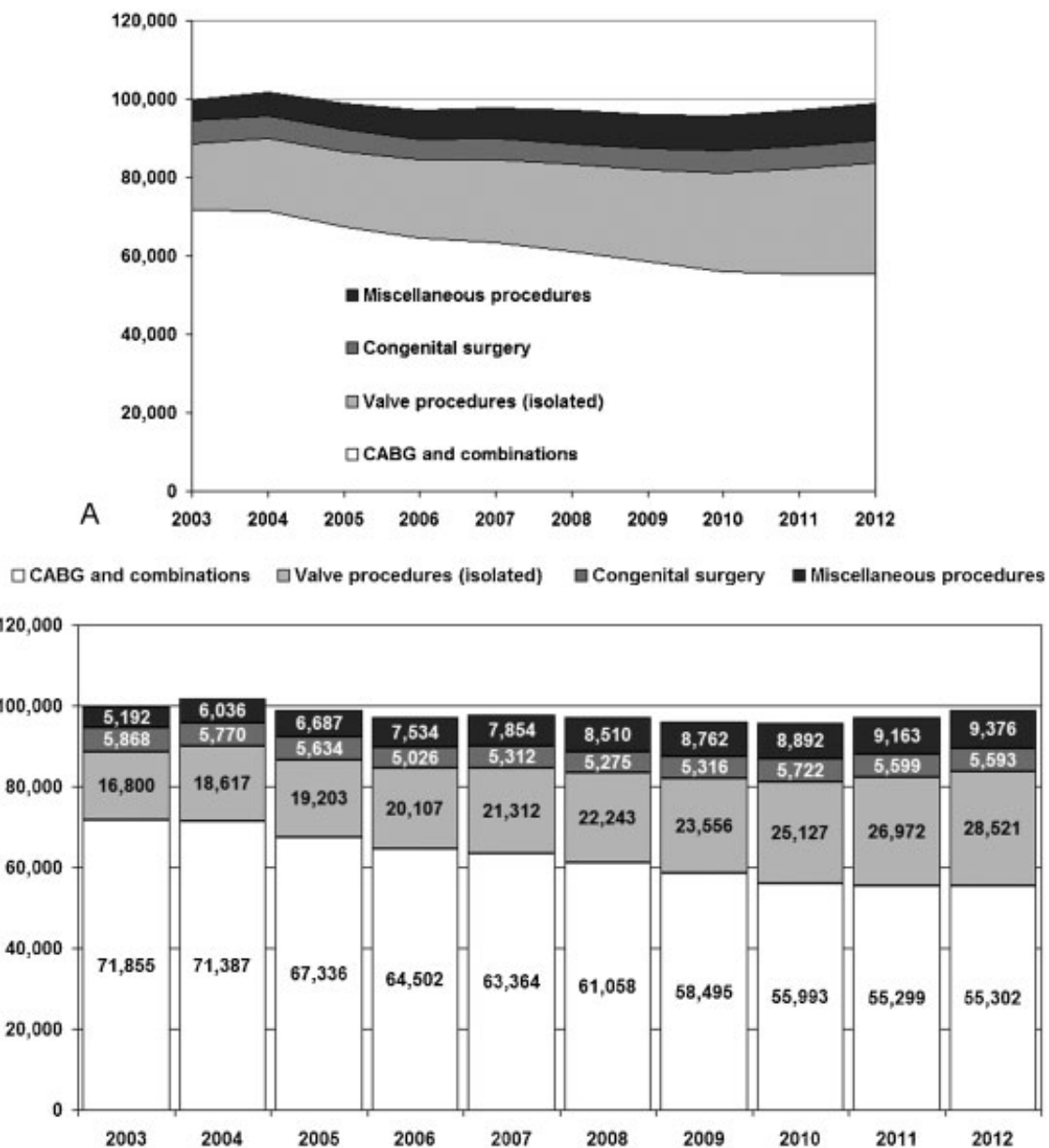

Fig. 1 (A) Cardiac surgery in Germany from 2003 to 2012. (1) CABG and combinations: all types of isolated coronary surgery with or without ECC and any combined procedure. (2) Valve procedures: all types of isolated heart valve surgery; heart valve procedures in combination with aortic surgery are summarized in the miscellaneous group. (3) Congenital heart surgery: all procedures with or without ECC; atrial septal defect repair in adults in combination with CABG or heart valve surgery are summarized in the CABG or heart valve surgery group. (4) Miscellaneous procedures: all other types of procedures with ECC. (B) Development of cardiac surgery in Germany during the past 10 years. CABG, coronary artery bypass grafting; ECC, extracorporeal circulation.

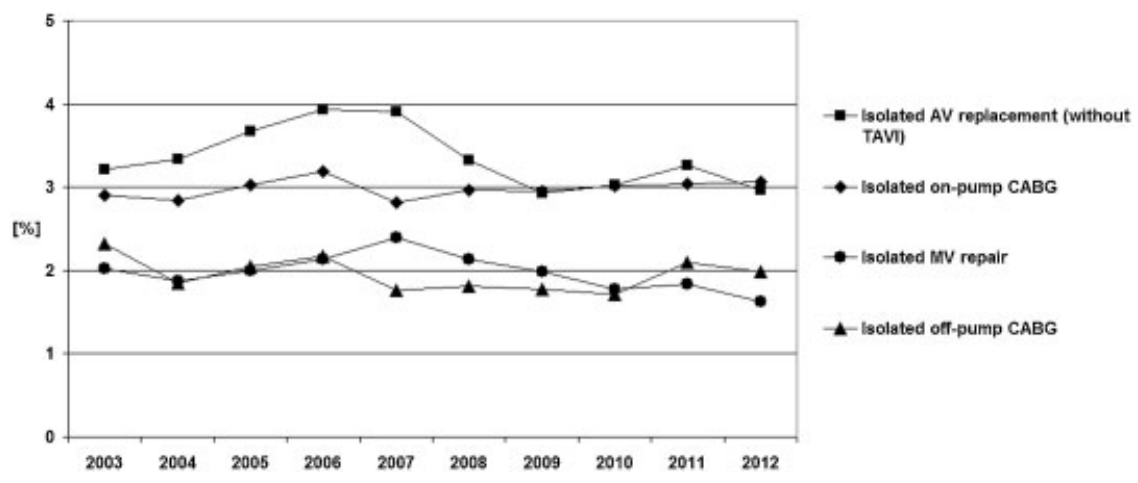

Fig. 2 Development of mortality for selected procedures. 
14 Cardiac Surgery in Germany during 2012 Beckmann et al.

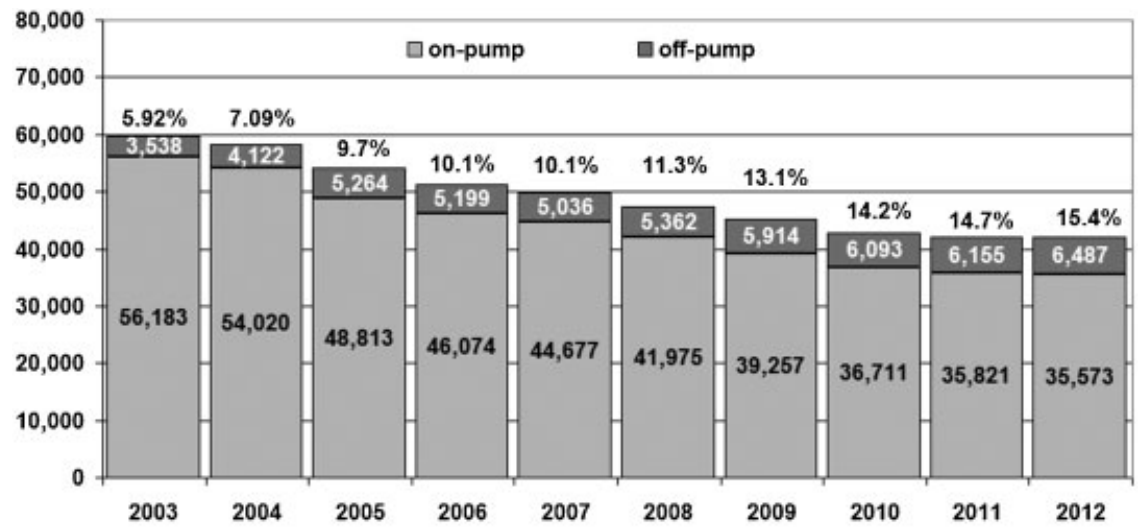

Fig. 3 Isolated CABG: the number of CABG declined since the year 2003 while the percentage of off-pump procedures slightly increased. CABG, coronary artery bypass grafting.

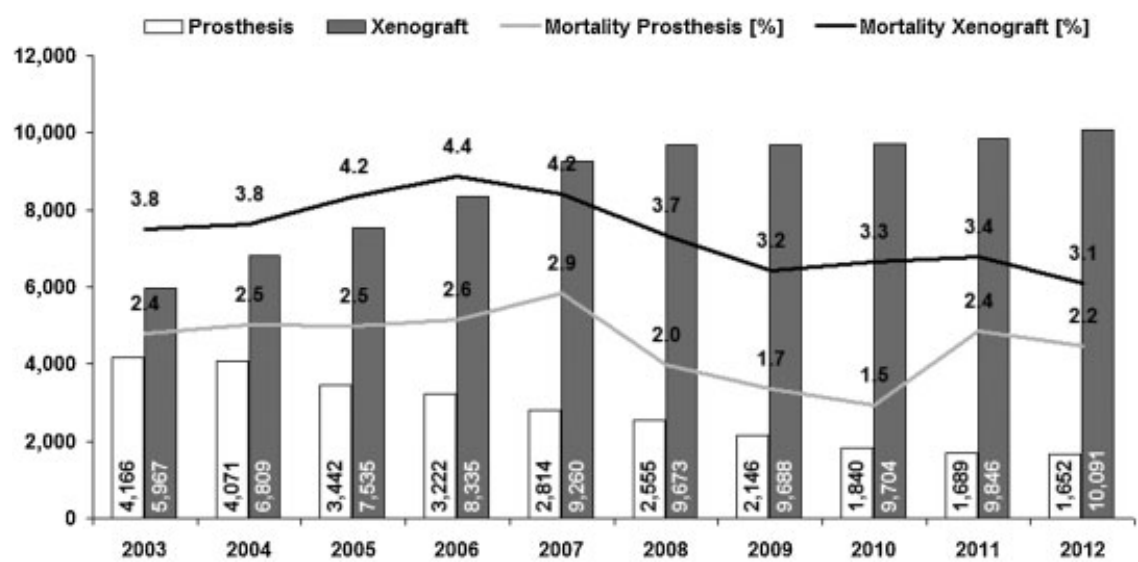

Fig. 4 Isolated aortic valve replacement from 2003 to 2012 in Germany. The use of xenografts continuously increased while the AVR using mechanical prostheses decreased. The sustained difference in mortality seems to be related to the difference in age pattern. Ross, homograft procedures, and TAVI are excluded in this overview. TAVI, transcatheter aortic valve implantation.

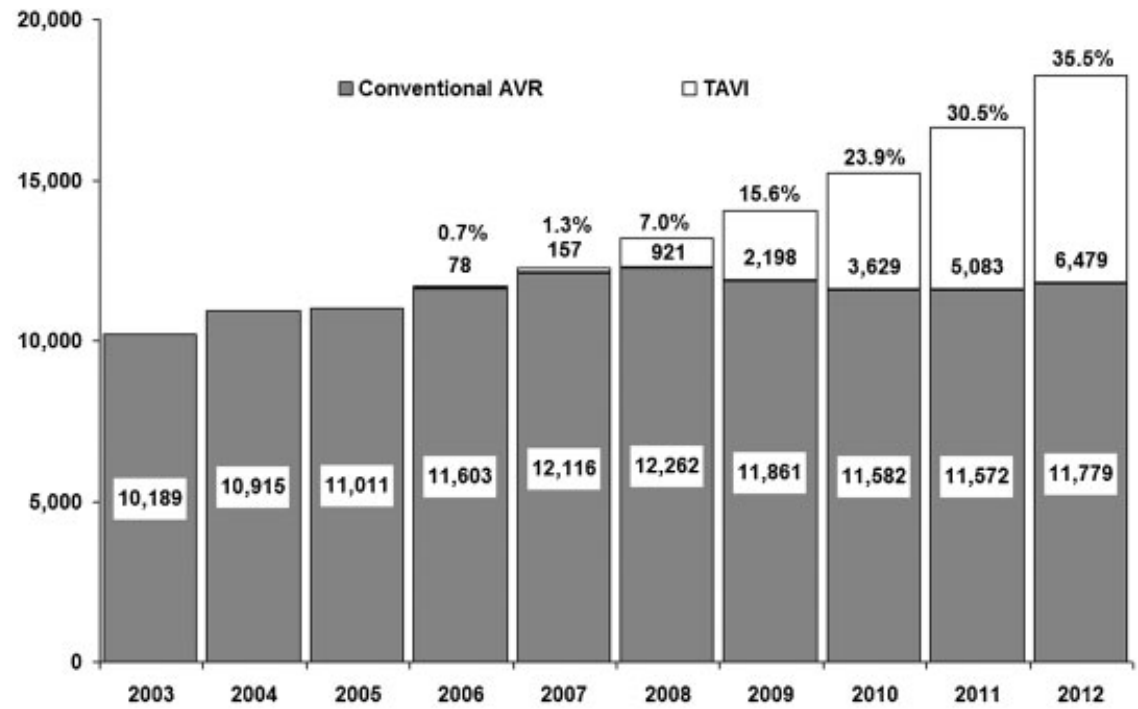

Fig. 5 Surgical aortic valve replacement or TAVI. The figure shows an obvious increase of TAVI. In 2012, 35.5\% of isolated aortic valve procedures were performed by endovascular or transapical implantation. TAVI, transcatheter aortic valve implantation. 


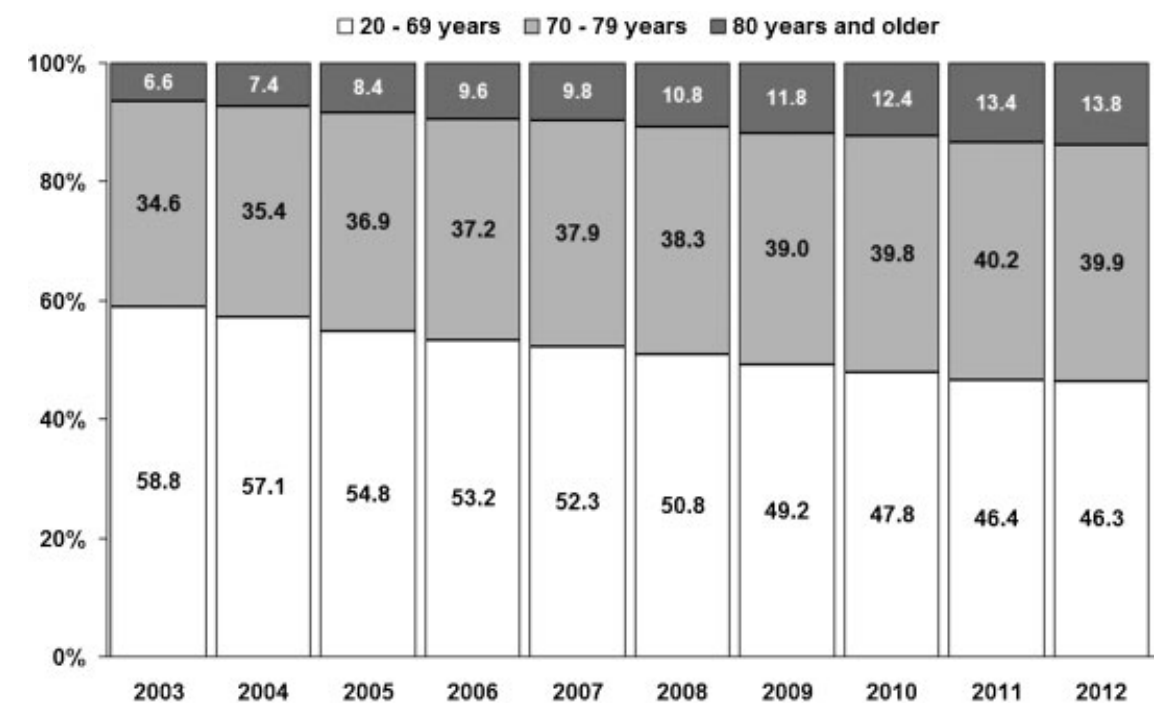

Fig. 6 Age distribution of cardiac procedures (ICD and pacemaker procedures excluded) since 2003. Currently, 53.7\% of the patients are at least 70 years. Patients younger than 20 years are excluded.

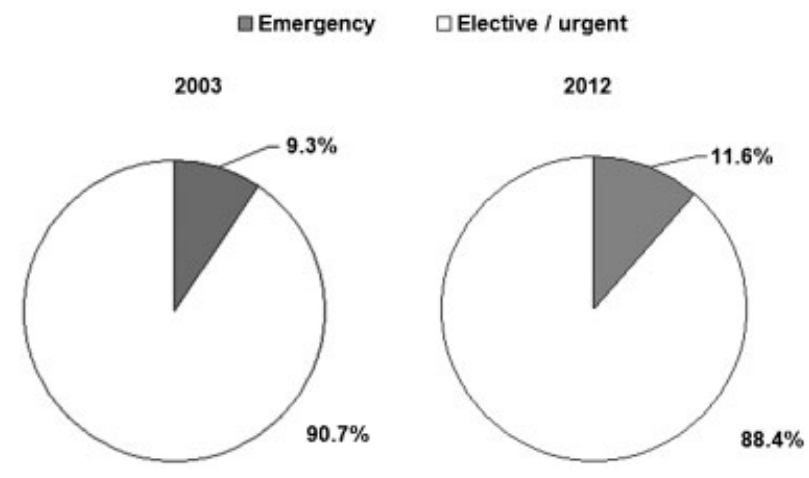

Fig. 7 Distribution of urgency 2003 and 2012.

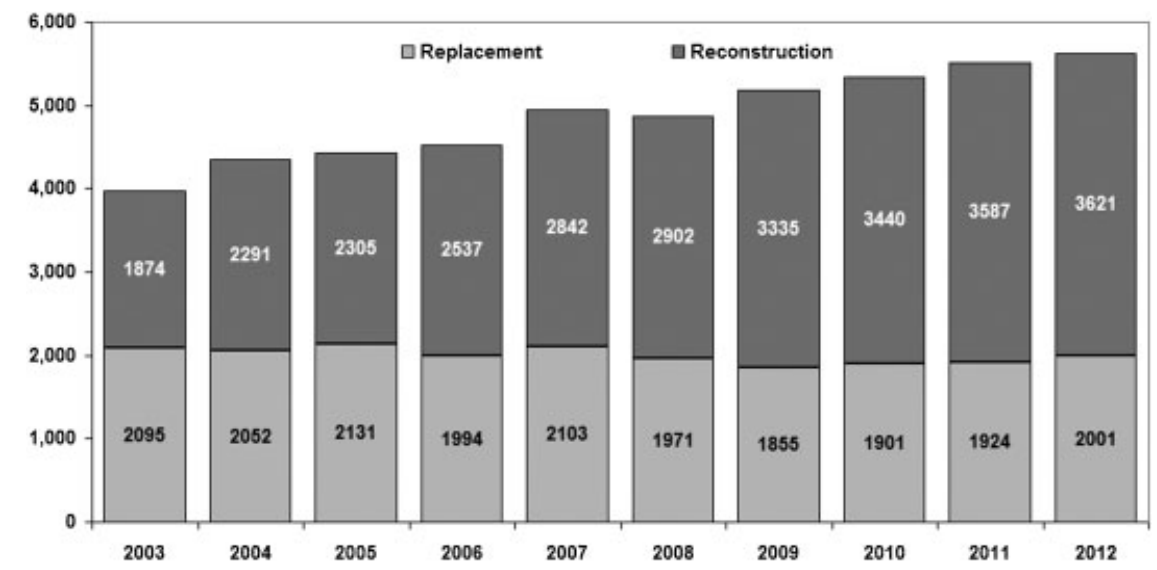

Fig. 8 Isolated mitral valve surgery over the past 10 years. In 64.4\% MV reconstructions and in 35.6\% MV replacements were performed. In 1994, the rate of reconstruction just reached $21 \%$. 


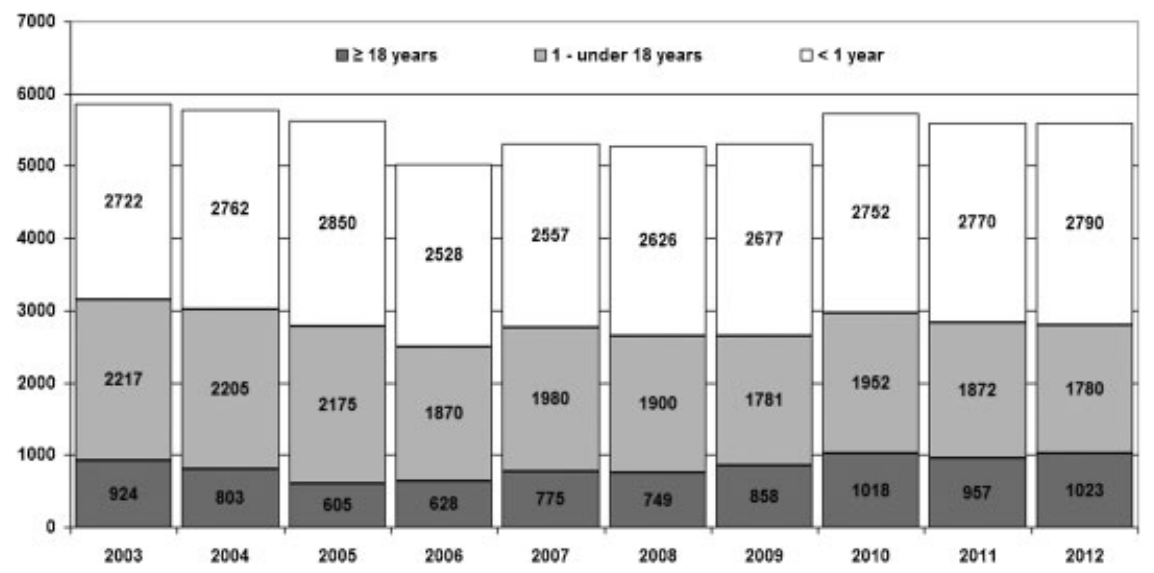

Fig. 9 Age distribution of patients undergoing congenital heart surgery in Germany over the past 10 years. No relevant changes can be observed. However, there may be a bias since not all relevant procedures can be allocated exactly to the congenital heart disease group in patients older than 18 years (e.g., aortic valve disease).

LVAD Implantation

BVAD Implantation

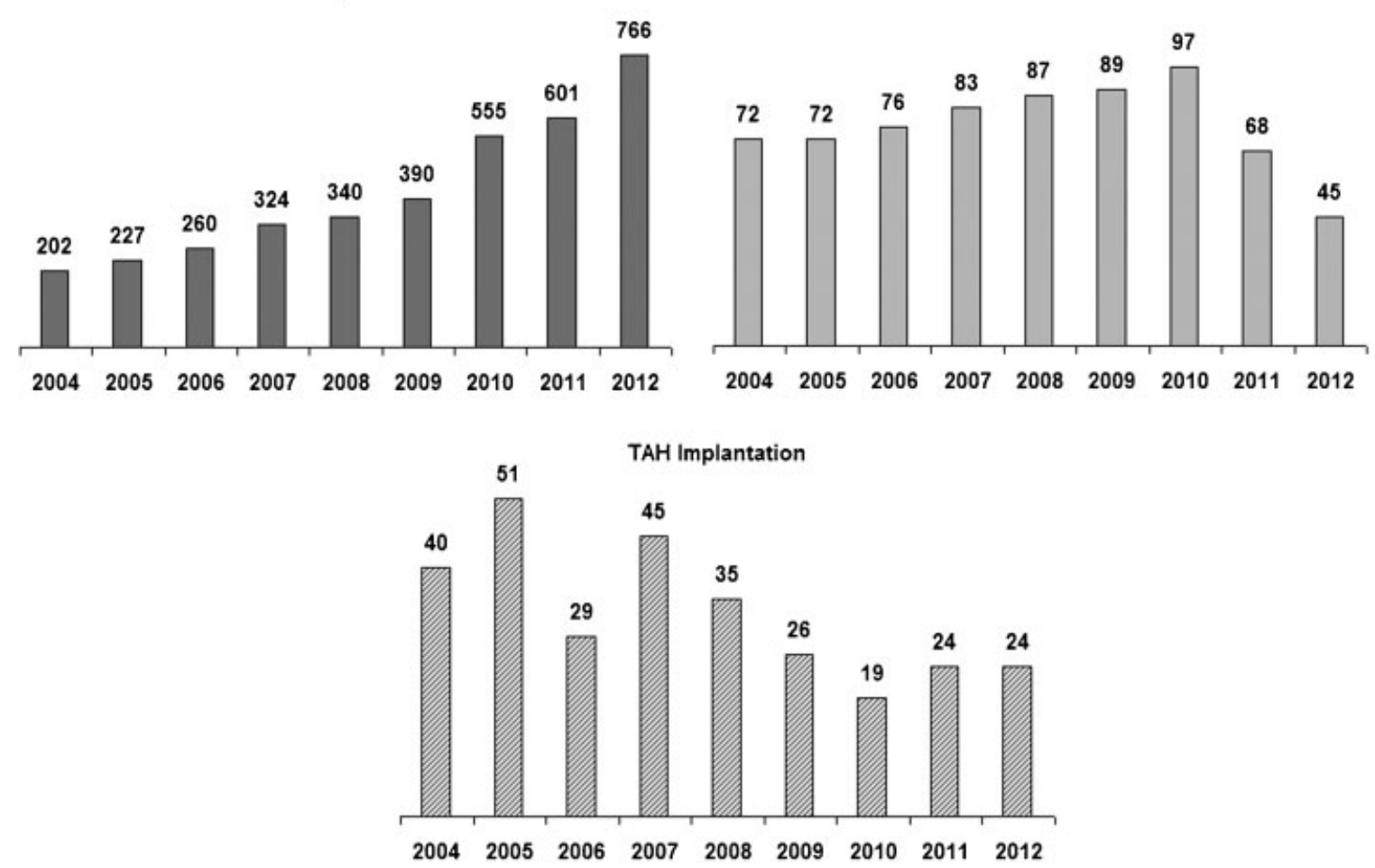

Fig. 10 Development of mechanical circulatory support in Germany over the past 9 years. There is a significant increase in implantations of left ventricular assist devices (LVAD). However, in 2012, the number of implanted paracorporeal biventricular support systems (BVAD) was only 66\% compared with the previous years. The number of total artificial heart (TAH) implantations remained on a low level.

\section{Acknowledgments}

On behalf of the German Society for Thoracic and Cardiovascular Surgery, the authors would like to thank the chairmen and their coworkers of all cardiac surgery units in Germany for their continued cooperation and support to realize this registry.

\section{References}

1 Kalmar P, Irrgang E. Cardiac surgery in the Federal Republic of Germany during 1989. A report by the German Society for Thoracic and Cardiovascular Surgery. Thorac Cardiovasc Surg 1990;38(3):198-200

2 Kalmar P, Irrgang E. Cardiac surgery in the Federal Republic of Germany during 1990. A report by the German Society for 
Thoracic and Cardiovascular Surgery. Thorac Cardiovasc Surg 1991;39(3):167-169

3 Kalmar P, Irrgang E. Cardiac surgery in Germany during 1991. A report by the German Society for Thoracic and Cardiovascular Surgery. Thorac Cardiovasc Surg 1992;40(3):163-165

4 Kalmar P, Irrgang E. Cardiac surgery in Germany during 1992. A report by the German Society for Thoracic and Cardiovascular Surgery. Thorac Cardiovasc Surg 1993;41(3):202-204

5 Kalmar P, Irrgang E. Cardiac surgery in Germany during 1993. A report by the German Society for Thoracic and Cardiovascular Surgery. Thorac Cardiovasc Surg 1994;42(3):194-196

6 Kalmar P, Irrgang E. Cardiac surgery in Germany during 1994. A report by the German Society for Thoracic and Cardiovascular Surgery. Thorac Cardiovasc Surg 1995;43(3):181-183

7 Kalmár P, Irrgang E. Cardiac surgery in Germany during 1995. A report by the German Society for Thoracic and Cardiovascular Surgery. Thorac Cardiovasc Surg 1996;44(3):161-164

8 Kalmár P, Irrgang E. Cardiac surgery in Germany during 1996. A report by the German Society for Thoracic and Cardiovascular Surgery. Thorac Cardiovasc Surg 1997;45(3):134-137

9 Kalmár P, Irrgang E. Cardiac surgery in Germany during 1997. A report by the German Society for Thoracic and Cardiovascular Surgery. Thorac Cardiovasc Surg 1998;46(5):307-310

10 Kalmàr P, Irrgang E. Cardiac surgery in Germany during 1998. A report by the German Society for Thoracic and Cardiovascular Surgery. Thorac Cardiovasc Surg 1999;47(4):260-263

11 Kalmár P, Irrgang E. Cardiac surgery in Germany during 1999. Thorac Cardiovasc Surg 2000;48(4):XXVII-XXX

12 Kalmár P, Irrgang E. Cardiac surgery in Germany during 2001: a report by the German Society for Thoracic and Cardiovascular Surgery. Thorac Cardiovasc Surg 2002;50(6):30-35

13 Kalmár P, Irrgang E; German Society for Thoracic and Cardiovascular Surgery. Cardiac surgery in Germany during 2002: a report by German Society for Thoracic and Cardiovascular Surgery. Thorac Cardiovasc Surg 2003;51(5):25-29

14 Kalmár P, Irrgang E; German Society for Thoracic and Cardiovascular Surgery. Cardiac surgery in Germany during 2003: a report by the German Society for Thoracic and Cardiovascular Surgery. Thorac Cardiovasc Surg 2004;52(5):312-317

15 Gummert JF, Funkat A, Krian A. Cardiac surgery in Germany during 2004: a report on behalf of the German Society for Thoracic and Cardiovascular Surgery. Thorac Cardiovasc Surg 2005;53(6): 391-399
16 Gummert JF, Funkat A, Beckmann A, Hekmat K, Ernst M, Krian A. Cardiac surgery in Germany during 2005: a report on behalf of the German Society for Thoracic and Cardiovascular Surgery. Thorac Cardiovasc Surg 2006;54(5):362-371

17 Gummert JF, Funkat A, Beckmann A, et al. Cardiac surgery in Germany during 2006: a report on behalf of the German Society for Thoracic and Cardiovascular Surgery. Thorac Cardiovasc Surg 2007;55(6):343-350

18 Gummert JF, Funkat A, Beckmann A, et al; German Society for Thoracic and Cardiovascular Surgery. Cardiac surgery in Germany during 2007: a report on behalf of the German Society for Thoracic and Cardiovascular Surgery. Thorac Cardiovasc Surg 2008;56(6): 328-336

19 Gummert JF, Funkat A, Beckmann A, et al. Cardiac surgery in Germany during 2008. A report on behalf of the German Society for Thoracic and Cardiovascular Surgery. Thorac Cardiovasc Surg 2009;57(6):315-323

20 Gummert JF, Funkat A, Beckmann A, et al. Cardiac surgery in Germany during 2009. A report on behalf of the German Society for Thoracic and Cardiovascular Surgery. Thorac Cardiovasc Surg 2010;58(7):379-386

21 Gummert JF, Funkat AK, Beckmann A, et al. Cardiac surgery in Germany during 2010: a report on behalf of the German Society for Thoracic and Cardiovascular Surgery. Thorac Cardiovasc Surg 2011;59(5):259-267

22 Funkat AK, Beckmann A, Lewandowski J, et al. Cardiac surgery in Germany during 2011: a report on behalf of the German Society for Thoracic and Cardiovascular Surgery. Thorac Cardiovasc Surg 2012;60(6):371-382

23 Gammie JS, Zhao Y, Peterson ED, O'Brien SM, Rankin JS, Griffith BPJ. J. Maxwell Chamberlain Memorial Paper for adult cardiac surgery. Less-invasive mitral valve operations: trends and outcomes from the Society of Thoracic Surgeons Adult Cardiac Surgery Database. Ann Thorac Surg 2010;90(5):1401-1408, e1, discussion 14081410

24 Vahanian A, Alfieri O, Andreotti F, et al; Joint Task Force on the Management of Valvular Heart Disease of the European Society of Cardiology (ESC); European Association for Cardio-Thoracic Surgery (EACTS). Guidelines on the management of valvular heart disease (version 2012). Eur Heart J 2012;33(19):2451-2496

25 Herbert MA, Prince SL, Williams JL, Magee MJ, Mack MJ. Are unaudited records from an outcomes registry database accurate? Ann Thorac Surg 2004;77(6):1960-1964, discussion 1964-1965 